\title{
White light intensity stabilization for long term photometric calibrations
}

\author{
João Cachatra ${ }^{1 *}$, David Alves ${ }^{1,2}$, Manuel Abreu ${ }^{1,2}$ \\ 1. Departamento de Física, Faculdade de Ciências, Universidade de Lisboa, Edifício C8, Campo Grande, PT1749-016 Lisbon, Portugal \\ 2. Instituto de Astrofísica e Ciências do Espaço, Universidade de Lisboa, Campo Grande, PT1749-016 Lisboa, Portugal
}

\begin{abstract}
This paper presents an overview of the design of a voice coil based white light intensity stabilization tool. A system is created capable of mechanically stabilizing the fluctuations in a beam of light. This is achieved with a use of a voice coil and simple PID based feedback loop, controlled using a LabVIEW interface. Stability results are shown using Allan deviations curves.
\end{abstract}

\section{Introduction}

The detection of planetary transits requires sensors with extremely high stability. The calibration of these sensors requires a light source with stability on par or better than the goal stability of the photometer.

As an example, the measurement of the flux impinging on the detector over extended periods of exposure, or the evaluation of the detector gain over time, require the probe beam to be extremely stable so that the relevant information in the signal can be retrieved within the intensity noise of the light source.

However, light sources found in most laboratories have nowhere near the stability that is required for the current generation of photometers used in astrophysics, particularly in the detection and characterization of planetary transits. Typical broadband light sources are listed as having 1-3\% fluctuations, or 10000 to 30000 ppm, for extended time periods. For example, the measurements of a Venus transit in a fixed wavelength mode and the relative variation in total irradiance require a precision of approximately 4 parts per million [1].

The main goal of this project is to develop a light unit and an intensity stabilization strategy in order to reduce its long term fluctuations, by at least one order of magnitude, so that it can be used in photometric calibrations.

\section{Luminous Power Control System}

Most high-precision light source procedures stabilize light at its source, meaning they try to directly control driving current and/or the temperature of the emitter source and circuitry. However, if access to the light source circuitry isn't possible a different method has to be used. Rather than trying to control primary source parameters like current or temperature, the intended system is based on the creation of an external occlusion system, meaning that intensity and stability of the light is modulated by an attenuator that obscures dynamically the beam of light as function of its variations at source level.

By creating a feedback system we can monitor the light intensity and with that information mechanically adjust the attenuator creating a shadow in the beam path, altering the net flux between the source and the exit port and thus reducing fluctuations in the beam.

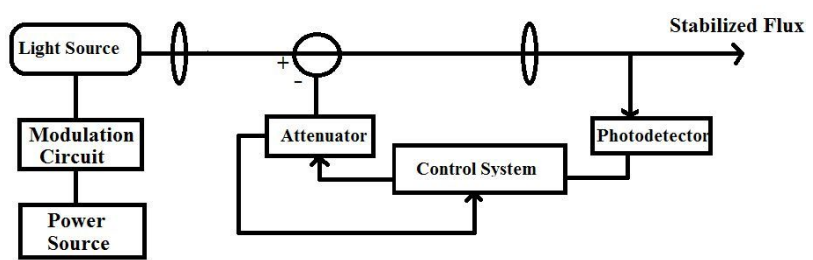

Figure 1: Basic block diagram of the system

The basic layout of this system consists of a photodetector that monitors the light intensity of the source, connected to a control system that tells the attenuator how much it needs to move to perform the necessary corrections.

\subsection{Light Source}

A current driven white light source is used to emulate a source with noise characteristics. This is achieved through a modulating circuit that connects the lamp to wave generator, enabling the modulation of the beam with a sinus waveform, and on a second stage, with a white noise waveform, mimicking a real light source. This approach allows me to have a good knowledge on the perturbations produced at light source level and thus a more controlled and accurate measure of the amount of ripple really corrected by the obscuration unit.

\subsection{Attenuator}

The attenuator used is a V-273 PIMag voice coil [2]. This voice coil drives consist of 2 essential components: a permanent magnet and a coil, which is located in the air gap of the magnetic field. When current flows through the coil, it moves in the magnetic field of the permanent magnet. The direction of motion depends on

Corresponding author: jlcachatra@gmail.com 
the polarity. Thanks to their low weight and friction-free drive principle, voice coil drives are particularly suitable for applications, which require high dynamics and high velocities at limited travel ranges.

\subsection{Detection and Control System}

The measurements from the photodiode are read using a Tektronix_DMM7510 digital multimeter. These readings are used to feed a PID control whose output determines the position of the voice coil. A LabVIEW interface is used to control and monitor the voice coil position and calculate the PID output. The PID gains were optimized using the Ziegler-Nichols tuning method [3].

\subsection{Experimental Results}

Several measurements were made to provide an overview of the systems capabilities. A long duration test, with a sampling rate of $50 \mathrm{mHz}$, was performed to assess the system's stability. The Allan deviation was chosen has the measurement for stability [4].

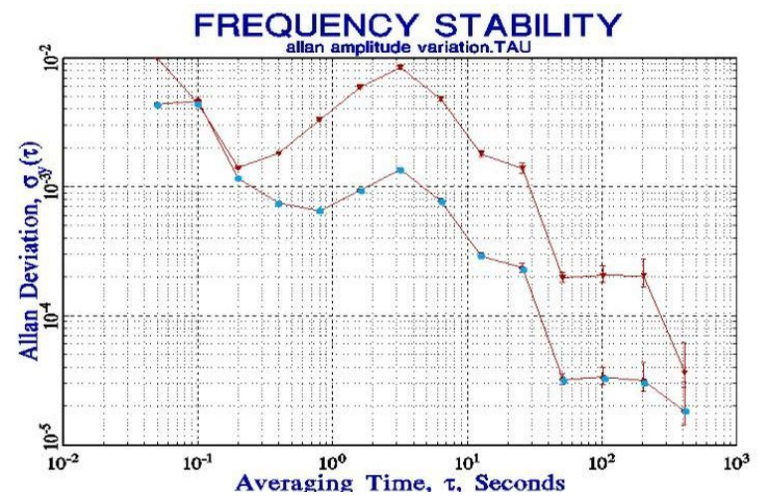

Figure 2: Allan deviation curves for a non-compensated (blue dots) and compensated (red dots) signal, with a frequency of $100 \mathrm{mHz}$ and an amplitude of $500 \mathrm{mV}$

Figure clearly shows the added stability provided by the system, increasing with the sampling time, reaching a minimum of $10^{-15}$. Further measurements were made to test the systems response to different frequencies and amplitudes of the modulating wave.

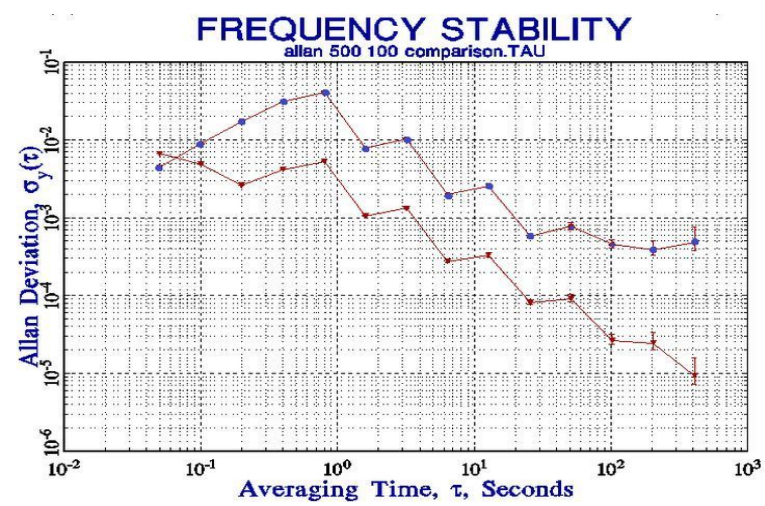

Figure 3: Allan deviation curves for compensated signals with a modulating frequency and amplitude of $300 \mathrm{mHz}$ (blue dots) and of $500 \mathrm{mHz}$ (red dots)
We can observe from figure 3 that the higher signal frequencies and amplitudes lead to a decrease in the achievable stability. A measurement was also made to test the system when subjected to a $100 \mathrm{mV}$ noise signal generated by the wave generator.

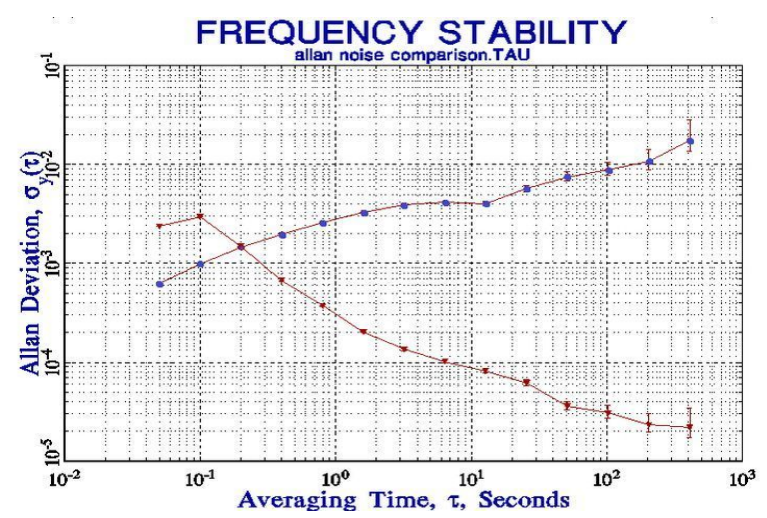

Figure 4: Allan deviation curves for a non-compensated (blue dots) and compensated (red dots) white noise signal, with an amplitude of $100 \mathrm{mV}$

Figure 4 shows how without compensation the system tends to become less stable the longer it runs, but when compensated the opposite occurs, becoming significantly more stable with time.

\section{Conclusions}

The development of this system requires a study of the noise sources present in the light source and the detection system used, the development of an optical collimating system and the development of a PID control system for the attenuator. Significant stability results are achieved when compared to the non-stabilized results.

This work was supported by Fundação para a Ciência e a Tecnologia (FCT) through the research grants UIDB/04434/2020 and UIDP/04434/2020. We also recognize the support of SPOF on the participation of this conference.

\section{References}

1. https://lasp.colorado.edu/home/sorce/2012/06/1 O/observations-of-the-venus-transit-totalirradiancel

2. https://static.physikinstrumente.com/fileadmin/u ser_upload/physik_instrumente/files/datasheets/ $V$-273-Datasheet.pdf

3. W. J. Riley, Handbook of Frequency Stability Analysis, NIST, (2007)

4. A. O'Dwyer, Handbook of PI and PID Controller Tuning Rules, Imperial College Press, 3, (2009) 